\title{
Fotografie und Deutschunterricht
}

\author{
Klaus Maiwald (Würzburg)
}

\section{Einleitung}

Als visuelles Medium steht die Fotografie einem Fach naturgemäß fern, dessen Gegenstände Sprache und Literatur sind. Nach landläufigen Vorurteilen sind Bilder vergröbernd und verkürzend, anstrengungs- oder gar gedankenlos aufnehmbar, oberflächlich, emotionalisierend und manipulierend. So gesehen steht Bildrezeption dem philologischen Anliegen der Durchdringung diskursiv verfasster symbolischer Ordnungen, wie sie Schrifttexte darstellen, diametral entgegen - und damit zumindest latent unter Verdacht.

Anders als Comics, Spielfilme und neuerdings Bildschirmspiele erzeugte die Fotografie in der Deutschdidaktik jedoch wenig Resonanz. Zwar trug sie im 19. Jahrhundert nicht unwesentlich zur Expansion visueller Illusionsbildung und damit zur Entprivilegierung der Literatur bei. Fotografie suggerierte eine magische Einheit des Zeigens mit dem Gezeigten, einen Schein von Anwesenheit und Präsenz (vgl. Hickethier 2001:43). Sie erforderte kein genialisches Künstlersubjekt, produzierte analoge Realitätsabbilder und läutete das Zeitalter massenhafter Reproduzierbarkeit ein (vgl. Hörisch 2004:233ff.). Andererseits erlangte sie alsbald selbst den Status einer hochkulturellen Kunstform und hatte, von Werbung und Propaganda vielleicht abgesehen, kaum Anteil an vermeintlich Kultur gefährdenden Verwerfungen, wie sie etwa das Fernsehen produziert(e). Im Gegenteil: In einer kommunikativ überhitzten Medienkultur wie der unseren erscheinen Fotos fast wie „befremdliche zenbuddhistische Verheißungen der Stille“ (Hörisch 2004:243). Möglicherweise haben diese Ambivalenzen dazu beigetragen, dass die Fotografie im deutschdidaktischen Diskurs wenig Aufmerksamkeit erfährt. Ein druckfrisches „Lexikon Deutschdidaktik“ (Kliewer / Pohl 2006) erwähnt sie im Zusammenhang mit anderen Stichwörtern, widmet ihr aber keinen eigenen Eintrag. 
In diesem Beitrag erfolgt zunächst eine knappe Bilanzierung bestehender Verbindungen zwischen Deutschunterricht und Fotografie. Etwas ausführlicher werden daraufhin weitergehende Möglichkeiten einer didaktischen Verzahnung aufgezeigt.

\section{Bestehende Verbindungen zwischen Fotografie und Deutschunterricht}

Fotografie und Deutschunterricht sehe ich in drei Bereichen verbunden: Erstens fungiert sie als Illustrationsmittel. Sichtbar wird dies v.a. in Lehrwerken, was ein Blick in Kennwort 8 (Hannover: Schroedel 1993), ein Sprachbuch mit einem besonders hohen Bildanteil, verdeutlichen kann. Fotografien erscheinen darin 1) als Beiwerk zu literarischen Texten (z.B. vom Autor) und zu Sachtexten (z.B. von einem Müllberg); 2) als Darstellungen verschiedener Kommunikationssituationen bzw. Sprachhandlungen (z.B. Protokollieren, Diskutieren, Interviewen); und 3) als Bestandteile genuiner Sachtexte.

Letzteres, das Foto in Sachtexten, ist eine eigene und wichtige Schnittstelle zwischen Fotografie und Deutschunterricht. Die Veröffentlichung der PISA-Studie (2001) hat Sachtexte nicht nur neu ins deutschdidaktische Bewusstsein gebracht (vgl. Hummelsberger 2003, Paule 2003), sie hat mit so genannten nicht-kontinuierlichen Texten (Tabellen, Bilder, Grafiken) auch daran erinnert, dass Sachtexte bildhafte Darstellungen enthalten bzw. selbst sein können. Neben fotografischen Abbildungen in Sachtexten gibt es weitgehend eigenständige Bild-Texte, etwa Karikaturen, Werbeplakate, „Pressefotos des Jahres“" oder Fotoromane in Jugendzeitschriften.

Eine Reihe von Verbindungen gibt es, drittens, zwischen Fotografie und Schreiben. $\mathrm{Zu}$ denken ist hier an die Bildbeschreibung i.e.S., aber auch an Fotografien als Vorlagen etwa für die Beschreibung eines Fahrrads, die Schilderung eines Rummelplatzes oder die Erzählung zu einer nächtlichen Tankstellenszene. Bilder können neben dem herkömmlichen „Aufsatzunterricht“ auch für kreatives Schreiben (vgl. im Überblick Spinner 2001) funktional werden, und zwar für gestalterisches / poetisches / literarisches Schreiben ebenso wie für ein stärker auf Selbsterfahrung zielendes, ,personalkreatives Schreiben“(vgl. Schuster 1997:103f., 158; Beisbart 2000:102; zum Schreiben zu Bildern Maiwald 2005:142-150; zu Bildern im Deutschunterricht allgemein Blei-Hoch 2006). Ich gebe ein Beispiel (Abb. 1): 
Abb. 1: Fotografie als Anregung für das Schreiben (Foto: Andreas Riedel, in: Haberkamm / Riedel 1999: 4). Aus urheberrechtlichen Gründen nicht enthalten.

Diese Fotografie könnte sowohl stärker gebundene Texte bzw. Schreibaufträge (Schilderung, Beschreibung) als auch freieres Schreiben anstoßen: Das Pferd erzählt seine Geschichte / Das Märchen vom Holzpferd / Ein „Holzpferd“-Elfchen / Was eines Nachts auf dem Dachboden geschah / Ein Schaukelpferd - zwei Leben(släufe) / Kindheit.

Als Illustrationen, als Bestandteile von Sachtexten und als Schreibanstöße verbinden sich Fotografien also mit dem Deutschunterricht. Geht man davon aus, dass Illustrationen in der Regel Beiwerk bleiben, dass der Deutschunterricht visuelle Anteile von Sachtexten immer noch gerne ausklammert und dass kreatives Schreiben in der schulischen Praxis wenig vorkommt, ergibt sich eine eher bescheidene Bilanz. Wie könnte das Ineinandergreifen von Fotografie und Text aus der Deutschdidaktik heraus weiter entwickelt werden? Die im Folgenden aufgezeigten Perspektiven sind nicht originär neu. Vielmehr soll deutlich werden, dass und wie bislang randständige bzw. rudimentäre Einbindungen der Fotografie in den Deutschunterricht auszubauen wären.

\section{Perspektiven didaktischer Weiterentwicklung}

\subsection{Fotografien in der Konkretisation literarischer Texte}

Um der Bedeutungsoffenheit und der Ästhetik literarischer Sinnmodelle gerecht zu werden, plädiert die Deutschdidaktik weithin gegen eine Verengung des Umgangs mit poetischen Texten auf deren diskursive Analyse. Vorgeschlagen werden affektorientierte „Lesarten“ (Abraham 1994), der Anschluss auch kreativen Schreibens an literarische Texte (z.B. Paefgen 1996, Waldmann 1999, Maiwald 2001) oder akustische, 
visuelle und szenische Gestaltungen (z.B. Haas 1997, Spinner 2002). Harald Frommer hat als literaturdidaktisches Prinzip den Gang „Von der Konkretisation zur Interpretation“ (1988) vorgeschlagen. Das heißt: Die Auseinandersetzung mit einem Text beginnt mit imaginativen Verstrickungen und vorläufigen, möglicherweise auch „,bornierten“" Deutungen. Aus solchen subjektiven Konkretisationen heraus (und nicht über sie hinweg!) werden dann intersubjektive Interpretationen entwickelt.

Für konkretisierende Annäherungen und Verstrickungen können auch Fotografien fruchtbar gemacht werden - zumal auch manche Künstler Kombinationen von Bild und Text vorführen. Denken lässt sich an Sophie Calle oder Jochen Gerz (vgl. Kreuzer 2005), an Ralf Schmerbergs Gedichtverfilmungen in „Poem“ (2003) (vgl. Hesse / Krommer / Müller 2005) oder an Fotografie-/Gedichtkombinationen, in denen Andreas Riedel (vgl. Abb. 1) und Helmut Haberkamm verschwindendes Dorfleben dokumentieren (1999). Gemeint ist aber primär, dass Leser Fotografien als Kontexte hinzuziehen. Georg Haarmann etwa ließ SchülerInnen in PowerPoint Kriegsgedichte mit Fotografien verbinden und so „eigenwillige Synästhesieeffekte [und] neue Interpretationswege" erzeugen (2002:52).

Hier ein weiteres Beispiel: Kombiniert man Hugo Balls „Karawane“ mit dem Foto einer Karawane (Abb. 2), so lässt dies auf eine literale Lesart des Textes schließen: Abb. 2: Fotografie als konkretisierender Kontext eines lyrischen Textes (aus einer Zulassungsarbeit von Ute Schlegelmilch, Universität Bamberg, 2004). Aus urheberrechtlichen Gründen nicht enthalten.

Der Schüler wollte in dem Gedicht die Stimmen und Rufe der Antreiber hören. In der Software Texte.Medien (Bekes / Frederking 2001), lässt sich „Karawane“ auch anders, quasi metaphorisch konkretisieren (Abb. 3): 


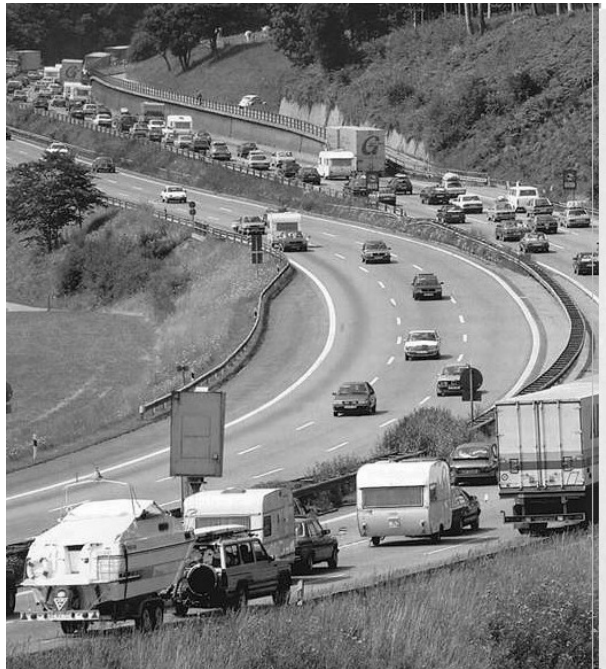

\section{KARAWANE}

jolifanto bambla $\hat{0}$ falli bambla

grossiga m'pfa habla horem

égiga goramen

higo bloiko russula huju

hollaka hollala

anlogo bung

blago bung

blago bung

bosso fataka

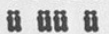

schampa wulla wussa ólobo

hej tatta gôrem

eschige zunbada

wulubu ssubudu uluw ssubudu

tumba ba- umf

kusagauma

ba - umf

Abb. 3: Fotografie als konkretisierender Kontext eines lyrischen Textes in Texte.Medien (1)

Texte.Medien ist ein interaktiver Assoziations- und Interpretationsraum (vgl. Berghoff 1997 und Schneider / Berghoff 2000), der es u.a. gestattet, Texte und Fotografien zu collagieren. So kann man Ernst Jandls „wien : heldenplatz“ auf eine historische Fotografie von 1938, aber auch auf einen völlig konträren Hintergrund legen (Abb. 4):

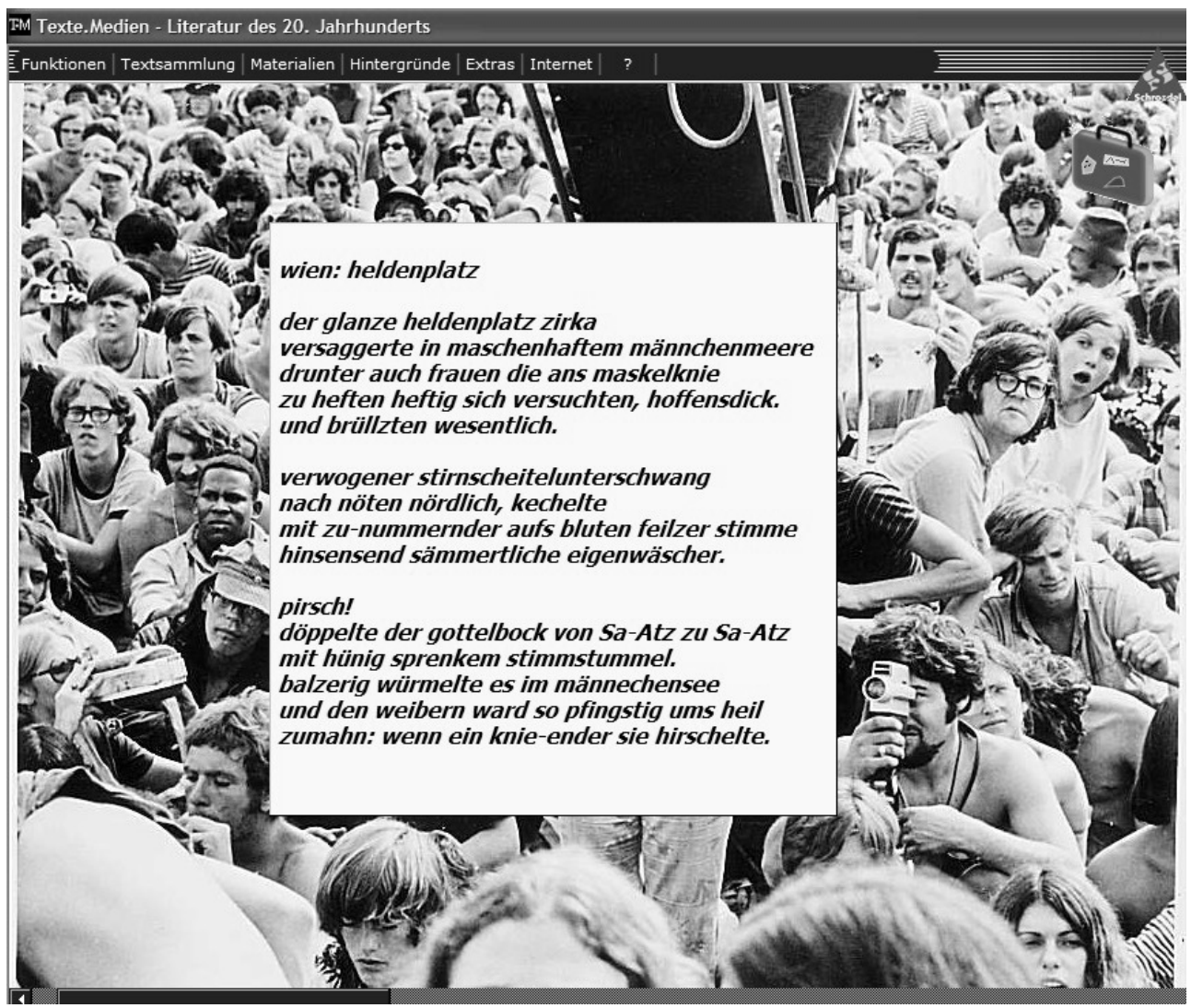

Abb. 4: Fotografie als konkretisierender Kontext eines literarischen Textes in Texte.Medien (2) 
Besonders im Zusammenspiel mit digitalen Gestaltungsmedien können Fotografien als visuelle Kontexte im Umgang mit Literatur also funktional werden. (Selbstverständlich kann Literaturunterricht dabei nicht stehen bleiben, sondern muss weiterführen zu intersubjektiver Reflexion und Interpretation.)

\subsection{Fotografien als Bildanteile von Sachtexten bzw. als „Bildtexte“}

Mit der so genannten kommunikativen Wende vollzog der Deutschunterricht bereits in den frühen 1970er Jahren auch eine (meist medien- und ideologiekritische) Hinwendung zu massen- und multimedialen Texten wie Comics, Zeitschriften, Werbeanzeigen. Heute wäre insbesondere im Umgang mit Sachtexten ein stärkeres Augenmerk auf deren visuelle Anteile zu richten. Ein von Martin Fix und Roland Jost 2005 herausgegebener Band führt die Vielfalt der Texte und der didaktischen Ansätze vor und leistet somit eine wichtige (Neu-)Positionierung im deutschdidaktischen Diskurs. Visuelle Elemente werden dabei aber nur in Ansätzen thematisiert (etwa in den Beiträgen von Vogt und Thielking, ebd.).

Nun würde eine auf lineare Schriftlichkeit verengte Sachtext-Didaktik gewiss zu kurz greifen. Was es heißen kann, eine Bildergänzung mit zu dekodieren, ihr Verhältnis zum Text zu bestimmen und eine Bewertung der Rhetorik und Pragmatik der entstehenden Bild/Text-Kombination vorzunehmen, zeigt das folgende Beispiel (Abb. 5):

Abb. 5: Fotografie und Sachtext (Quelle: Süddeutsche Zeitung, 13.01.2006, S. 5). Aus urheberrechtlichen Gründen nicht enthalten.

Im Januar 2006 erschien in der Süddeutschen Zeitung dieser Artikel über die brutale Misshandlung eines dunkelhäutigen Jungen durch Jugendliche in einem Dorf in Sachsen-Anhalt. Die eingebettete Fotografie zeigt ein breitbeinig dastehendes Paar schwarzer Springerstiefel. Ihr Träger steht auf Asphalt, vereinzelt liegen Blätter (?) oder Zigarettenkippen (?) herum. Seine schwarze Hose ist militärisch in die Stiefel gestopft, die Farbe der Schnürsenkel markiert ihn als mindestens rechtsgerichtet. Der emotionale Konnotationswert geht über das Abgebildete weit hinaus. In George Orwells Roman 1984 wird ein boot stamping on a human face als Schreckbild der Zukunft propagiert. In der Tat verbinden wir mit Stiefeln der gezeigten Art SS- und KZGräuel, Diktatur, Folter, glatzköpfige Neonazis. Somit weist die Fotografie die be- 
schriebene Tat als besonders abscheulich aus und die Täter einem rechtsradikalen Hintergrund zu.

Diese Bildbotschaft wird durch den Text freilich nicht vollständig gedeckt. Die Bildunterschrift sagt, dass das Opfer die Stiefel seiner Peiniger lecken musste; sie führt aber insofern in die Irre, als es sich dabei natürlich weder um die gezeigten Stiefel noch um den Träger handelte. Auch räumt der Text ein, dass ein rechtsradikaler Hintergrund nicht gesichert ist. (Die rechtsextreme Gruppe der Nachbarstadt wird damit zitiert, dass sie sich von den Tätern distanziert hat.)

Somit wäre diese Text-/Bildkombination differenziert zu bewerten: kritisch, weil das Foto den Sachverhalt nicht dokumentiert, sondern interpretiert. Auf der anderen Seite sind die klobigen Stiefel ein wirkungsvoller Blickfang und Leseanreiz. Vor allem aber sind sie eine weit eindringlichere Vergegenwärtigung der Leiden des Opfers als die bloße Rede davon. Überdies kontrastiert die abscheuliche Tat so scharf mit der beschriebenen Dorfidylle, dass das suggestive Bild angebracht scheint.

Wie der fotografische Anteil in die Arbeit mit einem Sachtext einbezogen werden kann, soll ein Beispiel (aus einer Lehrerfortbildung 2005) zeigen. Hierbei wurde ein Feuilleton-Text über den verschwenderischen Umgang mit Energieressourcen in den USA für den Abdruck in der Zeitung aufbereitet, u.a. durch Hinzufügung von Schlagzeile und Zwischenüberschriften. Überdies war aus zwei angebotenen Fotografien eine auszuwählen, mit einer Bildunterschrift zu versehen und die Entscheidung zu begründen. Zur Wahl stand ein Motiv aus einem Postkartenkalender, das einen neonerleuchteten Diner an der Route 66 mit davor geparktem Straßenkreuzer im Stil der 1960er Jahre zeigte. Die in der Zeitung tatsächlich verwendete Fotografie war die einer unberührt wirkenden Landschaft im US-Bundesstaat Idaho (Abb. 6):

Abb. 6: Fotografie zur Auswahl für die Illustration eines Sachtextes (Quelle: Süddeutsche Zeitung, 06.10.2005, S. 13). Aus urheberrechtlichen Gründen nicht enthalten.

Der Arbeitsauftrag erforderte eine genaue Lektüre des Textes, eine aufmerksame Betrachtung der Bilder und eine intensive Reflexion von Text-Bild-Bezügen. (Erweiter- 
bar wäre das Verfahren, indem man Bilder selbst suchen und die Texte am PC gestalten lässt.)

Auch der Werbung kommt man mit einem auf Schrift verengten Textbegriff nicht bei. Werbung hat sich in den letzten 20 Jahren auffällig von einer (eher verbalen)

Kaufrhetorik zu einer (stark visuellen) Erlebnisästhetik gewandelt (vgl. Maiwald 1999, Hurrelmann 2000, Marci-Boehncke 2006). Berücksichtigung findet dies z.B. in den mediendidaktischen Analysen verschiedener Jeans-Kampagnen von Wolfgang Gast (1994) oder in der ikonographischen Rekonstruktion des Werbemotivs der ,verzückten Nonne“ durch Thomas Bickelhaupt und Gerd Buschmann (2001).

Wie sehr Verbalsprache reduziert und Bildsprache aufgeladen sein kann, zeigt eine auf den ersten Blick wenig spektakuläre Werbeanzeige (Abb. 8):

Abb. 7: Fotografie als Werbetext (Quelle: Süddeutsche Magazin Nr. 12 vom 19.03.2004, S. 2). Aus urheberrechtlichen Gründen nicht enthalten.

Über die Eigenschaften der hier beworbenen Schuhe und Tasche (z.B. Preis, Material, Erhältlichkeit) sagt die Anzeige nichts, der verbale Anteil ist auf den Markennamen reduziert. Dagegen wird der Prestige- und Erlebniswert der Nobelmarke Hogan visuell überaus effektiv inszeniert. Das im original farbige Bild zeigt attraktive (lange, schlanke, braune) Beine einer Frau - auf dem Weg nach oben. Die mit Blüten bestreute Marmortreppe, der Fechter links und die Tempelsäule rechts wirken edel, mondän, kultiviert. Über ihren weißen Rock ist die Hogan-Trägerin farblich mit den visuellen Hochwert-Elementen verbunden. Der Mann im Hintergrund impliziert erotische Möglichkeiten, über allem lacht ein blauer Himmel, die Bewegungen gehen stimmig mit unseren Wahrnehmungspräferenzen nach rechts.

Beschreibung und Interpretation der Elemente und ihres Zusammenwirkens ergäben eine analytische Arbeit an diesem Bild; denkbar wären aber auch imaginative Fragestellungen: Welches Personenprofil geben wir der Frau? Was befindet sich am Ende der Treppe, wo streben die beiden hin, welche „Geschichte“ erzählt das Bild? Was wäre ein passender oder ironischer Slogan? Wie ließe sich Hogan per Bildbearbeitung zu einer Billigmarke umgestalten? 
Die Beispiele zeigen, dass Fotografien als Bestandteile von Sachtexten bzw. als eigenständige Texte gesteigerte Aufmerksamkeit verdienen. Sie zeigen auch, dass dies nicht rezeptiv-analytisch bleiben muss, sondern produktiv-kreative Elemente integrieren kann.

\subsection{Fotografien in der (kritischen) Medienreflexion}

Stärker heranzuziehen wären Fotografien auch für Medienreflexion und -kritik. Gegen die fotorealistische Euphorie standen früh bereits auch Sorgen über derealisierende Effekte des Mediums: Fotografie bannte Augenblicke, die in der Realität flohen, und sie machte fassbar, was mit bloßem Auge nicht zu fassen war. (Das klassische Beispiel sind Muybridges Serienaufnahmen eines galoppierenden Pferdes 1873/74; vgl. Hörisch 2004:232f.). Überdies erschloss sie - seit Röntgens Strahlen (1905) - vorher gänzlich ungesehene Wirklichkeiten.

Nun ist bereits der fotografische Normalfall qua Motiv und Perspektive stets ein Selektions- und Konstruktionsakt. Weil man Fotos obendrein retuschieren und mit Legenden versehen kann, öffnen sich weite Felder der Entwirklichung. Mit der Verbreitung digitaler Fotografie und Bildbearbeitung sind die Möglichkeiten der Verfälschung bzw. Virtualisierung fotografischer Realität enorm gestiegen. Denken lässt sich an Angela Merkels verschwundene Mundwinkel auf Wahlkampfplakaten oder an photoshop-gestylte Schönheiten in Illustrierten. Denken lässt sich auch an die Bilder, auf denen der so genannte tourist guy in alle möglichen Star-, Film- und Katastrophenszenen hineinmontiert war - und so zu einer kleinen Legende des Internet wurde. (Noch heute ergibt eine Bildsuche zu tourist guy über 600 Treffer.) Eines der Fotos war vom 11.09.2001 datiert und zeigte ihn auf der Aussichtsplattform des World Trade Center - samt anfliegender Verkehrsmaschine (vgl. http://urbanlegends.about.com/library/blphoto-wtc-g1.htm, 20.01.2006).

Man mag in der Bewertung solcher fakes geteilter Meinung sein, sollte aber nicht verkennen, dass sie nicht nur manipulatives und täuschendes, sondern auch subversives und kritisches Potenzial bergen. Nach dem Hurrikan „Katrina“ wurde 2005 - ebenfalls im Netz - folgende Fotomontage verbreitet (Abb. 9): 
Abb. 8: Fotografie / Fotomontage als subversive Kritik (Quelle:

http://www.donkeyontheedge.com/i/BushVaca.jpg, 20.01.2006). Aus urheberrechtlichen Gründen nicht enthalten.

Sohn und Vater Bush als Angler im überfluteten New Orleans waren eine beißende Kritik am unzulänglichen Krisenmanagement des U.S. Präsidenten und wiesen ihn (einmal mehr) als infantile Witzfigur aus. Im Deutschunterricht ließe sich mit diesem Bild arbeiten, indem man passende Legenden, Sprech- oder Gedankenblasen hinzufügt. (Der Originaltitel war Bush on vacation.) Weiter könnten Fotos dieser Art Reflexionen anstoßen über die Grenzen von Satire, die mediale Konstruiertheit von Wirklichkeit und die Medialität aller Wahrnehmung.

\subsection{Fotografie als Dokumentations- und Gestaltungsmedium}

Die genannten Verzahnungen von Fotografie und Text stehen in einem engen $\mathrm{Zu}$ sammenhang mit den Ideen eines medienintegrativen (Wermke 1997) oder ,symmedialen“ (Berghoff 1998:283) Deutschunterrichts. Medienintegration und Symmedialität bedeuten aber nicht nur eine Erweiterung des printmedialen Gegenstandsfeldes, sondern auch eine Veränderung des Lehrens und Lernens. Nicht nur erweitert sich also das Textkorpus, es verändern sich auch die Textumgangsformen: Sachtexte werden am PC gestaltet, Gedichte und Fotos in PowerPoint oder in Texte.Medien collagiert, Werbefotografien durch Bildbearbeitung ironisiert usw.

Die kostengünstige und kinderleichte Digitaltechnik hat der Fotografie die Aura des Elitären für Liebhaber und Experten genommen, sie hat dem Deutschunterricht aber ein ausgezeichnetes Lernmedium gegeben. Digitalfotografie unterstützt die Eigenproduktion von Medienangeboten wie Klassenzeitungen, Fotoromanen (vgl. Reuen 2005) oder Webseiten; sie öffnet der Veranschaulichung von Lerngegenständen neue Optionen, auch durch Stand- bzw. Bildschirmbilder von Spielfilmen, Videos, Computerspielen; und sie begünstigt die Dokumentation von Lernprozessen und -ergebnissen. Diese vielfachen Einsatzmöglichkeiten des Fotografierens im Deutschunterricht zeigen z.B. die von Mechthild Dehn u.a. (2004) vorgestellten Unterrichtseinheiten und die Netzdokumentationen von mir selbst durchgeführter Unterrichts- und Lehrprojek- 
te (vgl. http://www.uni-wuerzburg.de/germanistik/did/materialien/maiwald_webdokus/dokumentationen.html, 24.01.2006).

\section{Fazit und Ausblick}

Sehen lernt der Mensch von selbst, naturwüchsig ist das Verstehen von Bildern aber keineswegs. Vielmehr wird in einer zusehends visuell geprägten Kultur das „Lesen“ von Bildern eine Erziehungsaufgabe. Bekräftigt wird diese These auch durch deutschdidaktische Veröffentlichungen: über „Bilderwelten. Vom Bildzeichen zur CD-ROM“ (Franz / Lange 1999), zum „Hören und Sehen“ (Wermke 2001) oder zu „Filmdidaktik und Filmästhetik“ (Frederking 2006). Das Augenmerk liegt dabei jedoch meist auf AV- und Computermedien.

Der vorliegende Beitrag bilanzierte hingegen Brückenschläge zwischen Fotografie und Text und entwarf Perspektiven didaktischer Weiterentwicklung: Fotografie(n) in der Konkretisation literarischer Texte, als Bestandteile von Sachtexten, als Gegenstände (kritischer) Medienreflexion, als Gestaltungs- und Dokumentationsmedium für Lernprozesse und -produkte. Keine dieser Perspektiven enthält originär Neues, sehr wohl aber wichtige und fruchtbare Akzentuierungen für den Deutschunterricht.

Weil in Medienangeboten Bilder häufig mit Sprache kombiniert sind, vor allem aber, weil Verstehen ein sozialer und damit sprachlicher Prozess ist, kommt dem Deutschunterricht für den Erwerb visueller Kompetenz eine Leitfunktion zu. Andererseits sind „Bilderwelten“ mit philologischen Begriffen, Kategorien und Wahrnehmungsgewohnheiten nicht zu fassen. Sie erfordern Offenheit für und Neugier auf das, was sich in unserer Kultur an Visualität vollzieht. Sie erfordern aber auch bildästhetische Kompetenzen. Nun lassen sich DeutschlehrerInnen nicht nebenbei noch zu Bildwissenschaftlern oder Kunstpädagogen ausbilden. Angesichts des iconic turn sollten sie eine visuelle Grundbildung gleichwohl erwerben. Material dafür gibt es: Herrman K. Ehmers (1971) immer noch lesenswerter Aufriss „,visueller Kommunikation“; Christian Doelkers (1997) Instrumentarium für die Beschreibung und Klassifikation von Bildern; Franz-Josef Rölls (1998) Nachweis von (ur-)symbolischen und mythischen Sedimenten in populären Medien; Ralf Schnells (2000) „Geschichte und Theorie visueller Wahrnehmungsformen“; die Rubrik „Filmische Sehschule“ in der Zeitschrift 
Der Deutschunterricht (z.B. Fehr 2001); Klaus Maiwalds (2005) auf kultursemiotischer Grundlage konzipierte Deutschdidaktik bilddominierter Medienangebote.

Vielleicht hat aber auch dieser Beitrag bereits zeigen können, dass das Bild kein Teufelszeug und das Bildverstehen kein Hexenwerk ist. 


\section{Literatur}

Abraham, Ulf (1994): Lesarten - Schreibarten. Formen der Wiedergabe und Besprechung literarischer Texte. Stuttgart: Klett.

Abraham, Ulf / Beisbart, Ortwin / Marenbach, Dieter / Koß, Gerhard (2000): Praxis des Deutschunterrichts. 2. Aufl. Donauwörth: Auer

Abraham, Ulf / Bremerich-Vos, Albert / Frederking, Volker / Wieler, Petra (Hrsg.) (2003): Deutschunterricht und Deutschdidaktik nach PISA. Freiburg: Fillibach.

Beisbart, Ortwin (2000): Assoziatives Schreiben. In: Abraham / Beisbart / Marenbach / Koß, S. 101-103.

Bekes, Peter / Frederking, Volker (2001): Texte.Medien. Literatur des 20. Jahrhunderts. Hannover: Schroedel [CD ROM].

Belgrad, Jürgen / Niesyto, Horst (Hrsg.) (2001): Symbol. Verstehen und Produktion in pädagogischen Kontexten. Baltmannsweiler: Schneider.

Berghoff, Matthias (1997): Hypermedia als weitere Chance für den Deutschunterricht? Skizze eines interaktiven Assoziations- und Interpretationsraums im Internet zu Ernst Jandls „wien: heldenplatz“. In: Osnabrücker Beiträge zur Sprachtheorie 55, S. 172-185.

Berghoff, Matthias (1998): „Wenn ich die Lehrer für eine Sache nicht kriege, kann ich Schule nicht verändern...". Pädagogisch sinnvolle Nutzung der Neuen Medien setzt Schulentwicklung voraus. In: Frederking (Hrsg.), S. 279-301.

Bickelhaupt, Thomas / Buschmann, Gerd (2001): Eindeutig zweideutige Symbolik. Verzückung und Ekstase in einer Kraftstoffwerbung: Eine ikonographisch-ikonologische Interpretation. In: Belgrad / Niesyto (Hrsg.), S. 158-171.

Blei-Hoch, Claudia (2006): Bilder im Deutschunterricht. In: Kliewer / Pohl (Hrsg.) [im Druck].

Bogdal, Klaus-Michael / Korte, Hermann (Hrsg.) (2002): Grundzüge der Literaturdidaktik. München: dtv.

Dehn, Mechthild, u.a. (2004): Zwischen Text und Bild. Schreiben und Gestalten mit neuen Medien. Freiburg: Fillibach.

Doelker, Christian (1997): Ein Bild ist mehr als ein Bild. Visuelle Kompetenz in der MultiMediagesellschaft. Stuttgart: Klett-Cotta.

Ehmer, Hermann K. (Hrsg.) (1971): Visuelle Kommunikation. Beiträge zur Kritik der Bewußtseinsindustrie. Köln: DuMont Schauberg.

Fehr, Wolfgang (2001): Filmästhetik und Bildrhetorik im Erfolgskino: James Camerons „Titanic" (USA 1997). In: Der Deutschunterricht 53, H. 4, S. 79-84.

Fix, Martin / Jost, Roland (Hrsg.) (2005): Sachtexte im Deutschunterricht. Baltmannsweiler: Schneider Verlag Hohengehren.

Franz, Kurt / Lange, Günther (Hrsg.) (1999): Bilderwelten. Vom Bildzeichen zur CD-Rom. Baltmannsweiler: Schneider.

Frederking, Volker (Hrsg.) (1998): Verbessern heißt Verändern. Neue Wege, Inhalte und Ziele der Ausbildung von Deutschlehrer(innen) in Studium und Referendariat. Baltmannsweiler: Schneider.

Frederking, Volker (Hrsg.) (2006): Filmdidaktik - Filmästhetik. Jahrbuch Medien im Deutschunterricht 2005. München: kopaed.

Frommer, Harald (1988): Lesen im Unterricht. Von der Konkretisation zur Interpretation (Sekundarstufe I und II). Hannover: Schroedel.

Gast, Wolfgang (1994): Welche Jeans sind die besten? Mediendidaktische Analysen in den Werbeantworten von Levi's und Diesel. In: Diskussion Deutsch 140, S. 386-400.

Haarmann, Hans-Georg (2002): Die türme stehn in glutt. Zur Arbeit mit PowerpointPräsentationen. In: Praxis Deutsch 29, H. 175, S. 52-56.

Haberkamm, Helmut / Riedel, Andreas (1999): Lichd ab vom Schuß. Gedichte und Fotografien. Cadolzburg: ars vivendi.

Haas, Gerhard (1997): Handlungs- und Produktionsorientierter Literaturunterricht. Seelze: Kallmeyer. 
Hesse, Matthias / Krommer, Axel / Müller, Julia (2005): „Poem“ - Lyrikverfilmungen als Impuls für den Deutschunterricht. In: Deutschunterricht H. 3, S. 44-48.

Hickethier, Knut (2001): Film- und Fernsehanalyse. 3. Aufl. Stuttgart und Weimar: Metzler $(=\mathrm{SM}, 277)$.

Hörisch, Jochen (2004): Eine Geschichte der Medien. Von der Oblate zum Internet. Frankfurt: Suhrkamp.

Hummelsberger, Siegfried (2003): „Sachtext-Leser“ oder „Sach-Bearbeiter“ ? Wie sachdienlich ist die Rede von Sachtexten in eigener Sache? In: In: Abraham / Bremerich-Vos / Frederking / Wieler (Hrsg.), S. 330-346.

Hurrelmann, Bettina (2000): Nie waren sie wertvoller als heute. In: Praxis Deutsch 27, H. 163 , S. 16-25.

Kliewer, Heinz-Jürgen / Pohl, Inge (Hrsg.) (2006): Lexikon Deutschdidaktik. Baltmannsweiler: Schneider [im Druck].

Kreuzer, Stefanie (2005): Fotografie : Text. Die frühen visuell poetischen Arbeiten von Jochen Gerz. In: Der Deutschunterricht 57, H. 4, S. 25-37.

Maiwald, Klaus (1999): Werbung zwischen Kapital und Kult. Anmerkungen zur Veränderung eines Lerngegenstands. In: Literatur und Sprache - didaktisch (LUSD) 14, S. 7-22.

Maiwald, Klaus (2001): Literatur lesen lernen. Begründung und Dokumentation eines literaturdidaktischen Experiments. Baltmannsweiler: Schneider.

Maiwald, Klaus (2005): Wahrnehmung - Sprache - Beobachtung. Eine Deutschdidaktik bilddominierter Medienangebote. München: kopaed.

Marci-Boehncke, Gudrun (2006): Werbung. In: Kliewer / Pohl (Hrsg.) [im Druck].

Paefgen, Elisabeth Katharina (1996): Schreiben und Lesen. Ästhetisches Arbeiten und literarisches Lernen. Opladen: Westdeutscher Verlag.

Paule, Gabriela (2003): Sachtexte lesen und schreiben - Fächerverbindungen nutzen. In: Abraham / Bremerich-Vos / Frederking / Wieler (Hrsg.), S. 347-360.

Reuen, Sascha (2005): Fotoromane im Deutschunterricht. In: Der Deutschunterricht, 57, H. 4, S. 77-84.

Röll, Franz-Josef (1998): Mythen und Symbole in populären Medien. Der wahrnehmungsorientierte Ansatz in der Medienpädagogik. Frankfurt / Main: Gemeinschaftswerk der evangelischen Publizistik.

Schneider, Olaf / Berghoff, Matthias (2000): Texte in digitalen Erfahrungsräumen. Zur Konzeption einer eigenaktiven, konstruktiven und kommunikativen Lernumgebung im Internet. In: Computer und Unterricht 39, S. 54-57.

Schnell, Ralf (2000): Medienästhetik. Zu Geschichte und Theorie visueller Wahrnehmungsformen. Stuttgart: Metzler.

Schuster, Karl (1997): Das personal-kreative Schreiben im Deutschunterricht (2. Aufl.). Baltmannsweiler: Schneider.

Spinner, Kaspar (2001): Kreatives Schreiben. In: Ders.: Kreativer Deutschunterricht. Seelze: Kallmeyer, S. 108-125.

Spinner, Kaspar H. (2002): Handlungs- und produktionsorientierter Literaturunterricht. In: Bogdal / Korte (Hrsg.), S. 247-257.

Thielking, Sigrid (2005): Ganz bei der Sache sein. Vom didaktischen Umgang mit Literaturgeschichte(n) als Sachtext(en). In: Fix / Jost (Hrsg.), S. 160-170.

Vogt, Rüdiger (2005): Über Textrahmen - am Beispiel von Tageszeitungen. In: Fix / Jost (Hrsg.), S. 133-149.

Waldmann, Günter (1999): Produktiver Umgang mit Literatur im Unterricht (2. Aufl.). Baltmannsweiler: Schneider.

Wermke, Jutta (1997): Integrierte Medienerziehung im Fachunterricht. Schwerpunkt: Deutsch. München: kopaed.

Wermke, Jutta (Hrsg.) (2001): Hören und Sehen: Beiträge zu Medien- und Ästhetischer Erziehung. München: kopaed. 